\title{
Current Saturation in Field Emission from H-passivated Si Nanowires
}

\author{
ACS-Nano vol. 6 no. 8 7463-7471 (2012)
}

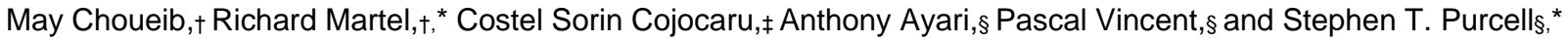 \\ †Département de chimie, Université de Montréal, Montréal, Québec H3T 1J4, Canada, \\ ‡Ecole Polytechnique LPICM, UMR 7647, F-91128 Palaiseau, France, and \\ §Laboratoire de Physique de la Matière Condensée et Nanostructures, Université Lyon 1, CNRS, UMR 5586, F-69622 \\ Villeurbanne, Cedex, France
}

\begin{abstract}
This paper explores the field emission (FE) properties of highly crystalline Si nanowires (NWs) with controlled surface passivation. The NWs were batch-grown by the vapor_liquid_solid process using Au catalysts with no intentional doping. The FE current_voltage characteristics showed quasi-ideal current saturation that resembles those predicted by the basic theory for emission from semiconductors, even at room temperature. In the saturation region, the currents were extremely sensitive to temperature and also increased linearly with voltage drop along the nanowire. The latter permits the estimation of the doping concentration and the carrier lifetime, which is limited by surface recombination. The conductivity could be tuned over 2 orders of magnitude by in situ hydrogen passivation/desorption cycles. This work highlights the role of dangling bonds in surface leakage currents and demonstrates the use of hydrogen passivation for optimizing the FE characteristics of Si NWs.
\end{abstract}

KEYWORDS: field emission . nanowires . semiconductor . surface states .hydrogen passivation

Under the right conditions, the field emission (FE) current_voltage characteristics from semiconductors can be radically different from those predicted by standard Fowler_Nordheim (FN) theory. Instead of the exponential FN law expectedfor metals, the emission current, I, can exhibit a saturation region as a function of applied voltage, V, that is more or less welldefined depending on a host of parameters such as the doping level and type, emitter temperature (T), crystal quality, and the state of the surface.Wehave recently shown such saturation phenomena for the first time for batch-fabricated, individual semiconducting (SC) SiC nanowires (NWs).1,2 The saturation was stable in current and extended over a wide range of voltages, which should tend to make uniform the emission of planar FE cathodes composed of a multitude of NWs randomly distributed over a surface. The current at saturation presented also a sharp photothermal sensitivity, which is of interest for laser- and heatcontrolled electron sources and light detectors. The cross-breeding of rich phenomena in SC NWs with FE principles thus appears promising for applications, but the parameters relating the emission properties of SC NWs to their electrical transport and surface properties are still poorly understood.

For FE, the saturation occurs theoretically and experimentally when the SC material is p-doped, though in principle it could also occur for prohibitively low n-type carrier concentrations. It manifests itself in strongly nonlinear FN plots, that is to say plots of $\log \left(\mathrm{I} / \mathrm{V}_{2}\right)$ versus $(1 / \mathrm{V})$. A first qualitative explanation for this behavior was given by Arthur, 3 and later a theoretical model was developed by Baskin.4 This model describes how the emission is controlled by a depletion zone near the emission end, as presented in Figure 1a. A slightly modified version of this model provides, however, the best explanation for the three different current_voltage [I(V)] regimes identified in Figure 1b: In the low-field region (region I), the emission is supplied by minority carriers coming from the conduction band. The I(V) follows a quite linear FN curve because increasing external field at the NW apex must correct for only a small resistive voltage drop in the SC compared to the total applied voltage. In the medium-field region (region II), the field is strong enough to induce an inversion layer (n-type) at the apex. Increasing the voltage further leads to a deeper penetration of the field into the emitter because of the limited carrier concentration, giving rise to a high-resistance space charge region (SCR) between the $p$-type bulk and the $n$-type surface,3 similar to a $p \_n$ junction in reverse bias (Figure 1a). The large associated internal voltage drops, $\Delta \mathrm{V}$, are directly measurable with an electron energy analyzer.1_3 This saturation regime is the most interesting because the current is, to a large degree, controlled by generation_recombination (GR) production of carriers and as a result is highly sensitive to temperature and light absorption. The current density is lower than for metal emitters, but the current is much more stable. This is 
in part because the $\mathrm{p} n \mathrm{n}$ junction acts as a series load that increases the internal voltage and stabilizes the emission. In addition, covalent materials such as Si are much less prone to gas absorption, which is the primary cause of FE instability. Finally, the less studied region III has been attributed to avalanche phenomena where the internal field becomes large enough for impact ionization in the SCR. This interpretation is, however, revisited here in the context of Si NW emitters at low doping concentration.

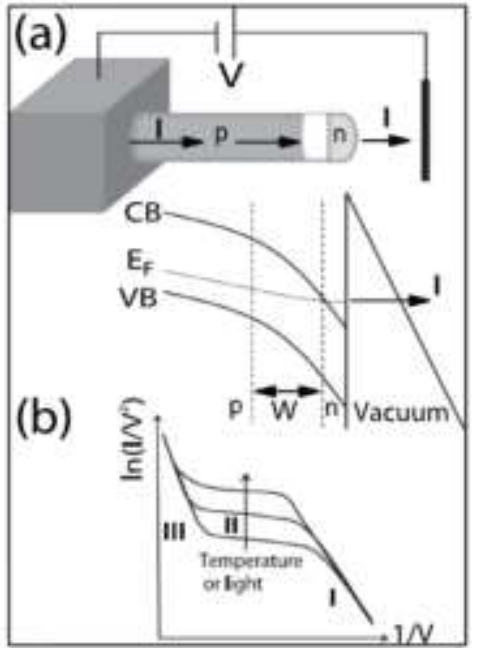

Figure 1. Internal $p \_n$ junction in a p-type nanowire during field emission: (a) schemas of the emission geometry and the potential energy diagram in the near surface region. A depletion region $(\mathrm{W})$ builds up behind a pocket of electrons ( $n$ ) formed in the degenerate conduction band. (b) Schema of the theoretical Fowler_Nordheim plots depicting the three current regimes and the effects of temperature or light.

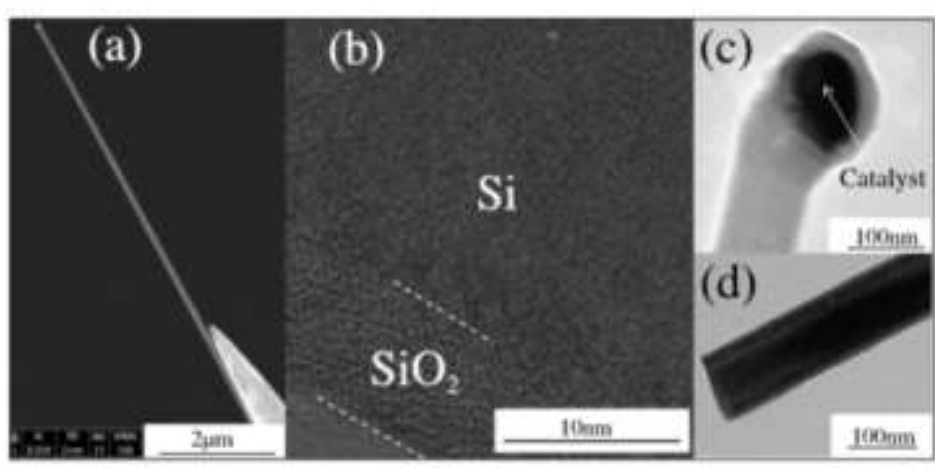

Figure 2. (a) SEM image of a SiNWwith diameter $d=100 \mathrm{~nm}$ and length $L=9 \mu \mathrm{m}$ attached to a tungsten tip. (b) TEM image showing the high crystallinity of the NW and the $10 \mathrm{~nm}$ oxide. (c) Catalyst at the NW end. (d) Low-resolution TEM image of the substrate end of the nanowire without catalyst. Note that TEM images of NW ends are often poorly resolved because NWs vibrate under the electron beam.

Despite the richness of FE from SCs, the corresponding literature is much less extensive than that devoted to emission from metals. This is because a successful observation of these regimes depends on special sample preparation. Previous studies on individual tip emitters fabricated manually from bulk SCs and prepared by high-field evaporation 3,5 and on Si tip arrays fabricated by lithography 6 revealed saturation effects. Saturation phenomena have been observed at low temperature $(\sim 100 \mathrm{~K})$ with reduced effects at room temperature. The saturation current density was 2 or 3 orders of magnitude larger than in a $p \_n$ junction, which was attributed to a leakage current. This current can originate from a surface inversion layer, a high density of bulk defects, surface states, etc. As well, the high temperature treatments needed to produce clean emitters often create irreversible surface changes which wash out the saturation effects.7 Consequently, the dynamic range over which I could be modulated by heat or light was reduced. Outside the scope of this article, heavy additional technologies were used to control the FE current by gated fieldss and pin junctions g fabricated by multistep lithography.

For NWs, the problem of surface states and defects is exacerbated because of the high surface-to-volume ratio. This explains why the few measurements in the literature on SC NWs have only shown standard FN behaviors.10_13 However, our previous results displayed somewhat muted saturation effects due to defects in the SiC NWs, which included different polymorphs, many dislocations, and uncertain surface quality.1,2 Moreover, saturation was observed, over a large background of leak current, only after the laborious deployment of low-energy Ar bombardment to remove surface contaminants. In this work, we present results on Si NWs of much higher structural quality grown using the vapor_solid_liquid (VLS) process. These displayed much stronger saturation regimes immediately and systematically after minimal heating in vacuum. Results of different experiments are presented that include measurements of $\mathrm{I}(\mathrm{V}, \mathrm{T})$ curves and total energy distributions (TED) during FE in ultrahigh vacuum (UHV). An exciting method to reversibly control the surface state density of Si NWs by in situ cyclic heating and hydrogen passivation is described.

\section{RESULTS}

This FE study was carried out on five individual NWs attached to the ends of tungsten tips (Figure 2a) (see Experimental Section). Scanning Electron Microscopy (SEM) images of these NWs showed very uniform diameters, d, of around $100 \mathrm{~nm}$ and lengths, L, up to $50 \mu \mathrm{m}$. Transmission Electron Microscopy (TEM) studies have demonstrated that the NW cores are high-quality single crystals of silicon covered by an amorphous, probably oxide, layer of about 
$10 \mathrm{~nm}$ (Figure 2b). No evidence of stacking faults was found. The oxide layer appears to be formed during the VLS NW growth (see Experimental Section). FE measurements were carried out in a dedicated UHV FE system that allowed in situ hydrogen passivation (see Experimental Section). The measurements on all five NWs studied so far were reproducible and consistent. Only two NWs are presented here for comparison: (1) NW1, Figure 2c, emission from the Au catalyst end $(L=20 \mu \mathrm{m}, d=100 \mathrm{~nm})$; (2) NW2, Figure $2 \mathrm{~d}$, emission from the substrate end $(L=40 \mu \mathrm{m}, \mathrm{d}$ $=100 \mathrm{~nm}$ ). The TEM image of the apex of NW1 (Figure 2c) shows the Au catalyst particle at the apex, as typically found in samples grown by the VLS tip growth mechanism. Figure 2d shows the substrate end of NW2 after it was broken off from the substrate along a transverse cleavage plane. There is no trace of the catalyst in this case. The high crystalline quality, the absence of structural defects, and the oxide passivation are key elements explaining the good electrical properties of these NWs. First measurements were carried out on the Si NWs without any treatment. These showed fairly straight FN plots due to strong leakage currents through an undefined physisorbed layer formed on exposure to ambient conditions. A 2 min heating to about 500 _ was used to remove the physisorbed contaminants. All samples after such in situ heat treatments exhibited highly nonlinear FE I(V) characteristics with strong current saturation, as predicted by the FE theory from p-doped SCs. Although the growth conditions for these Si NWs include no dopant, these results clearly indicate that the NWs were p-doped. This unintentional doping is ascribed to Au atoms or clusters deposited from the Au catalyst particles within the NWs during the growth. Indeed, it was shown that Au can induce p-doping 14 in addition to deep levels 15 in Si NWs. As discussed hereafter, the quasiideal FE characteristics observed from as-prepared Si NWs also indicated good passivation of the $\mathrm{Si}_{/} \mathrm{SiO}_{2}$ interface by the hydrogen treatment performed during growth.

Typical FN plots with increasing T are given in Figure 3a for NW1 and in Figure 3b for NW2. The corresponding I(V) curves are presented as semilog plots in the insets. The three regions predicted by theory are well-defined. The standard FN region I is at low current $(<1 \mathrm{pA})$ and is best seen in the single electron counter measurements of NW1. The current goes through very flat saturations in region II for both NWs. Although it is somewhat flatter and at lower current for NW2, this means that both NWs present low leakage currents. Region III starts at the end of region II with the transition voltage $\left(\Delta \mathrm{V}_{\mathrm{t}}\right)$ increasing with $\mathrm{T}$. There are no equivalent strong saturation effects found for SC NWs in the literature, and the curves are even flatter than almost all emitters obtained for bulk SC materials. Comparing these results quantitatively with the literature for macroscopic emitters is rather difficult because one must develop a correct scaling to allow for the varying emitter areas, conic or linear shapes, applied voltages, field amplification factors, and temperatures. However, note that a strong saturation is found here even at room temperature, while in most work, this could be achieved only by cooling. The strong saturation must be related to the low defect and surface state densities at the $\mathrm{Si} / \mathrm{SiO}_{2}$ interface due to hydrogen treatments during the NW growth.
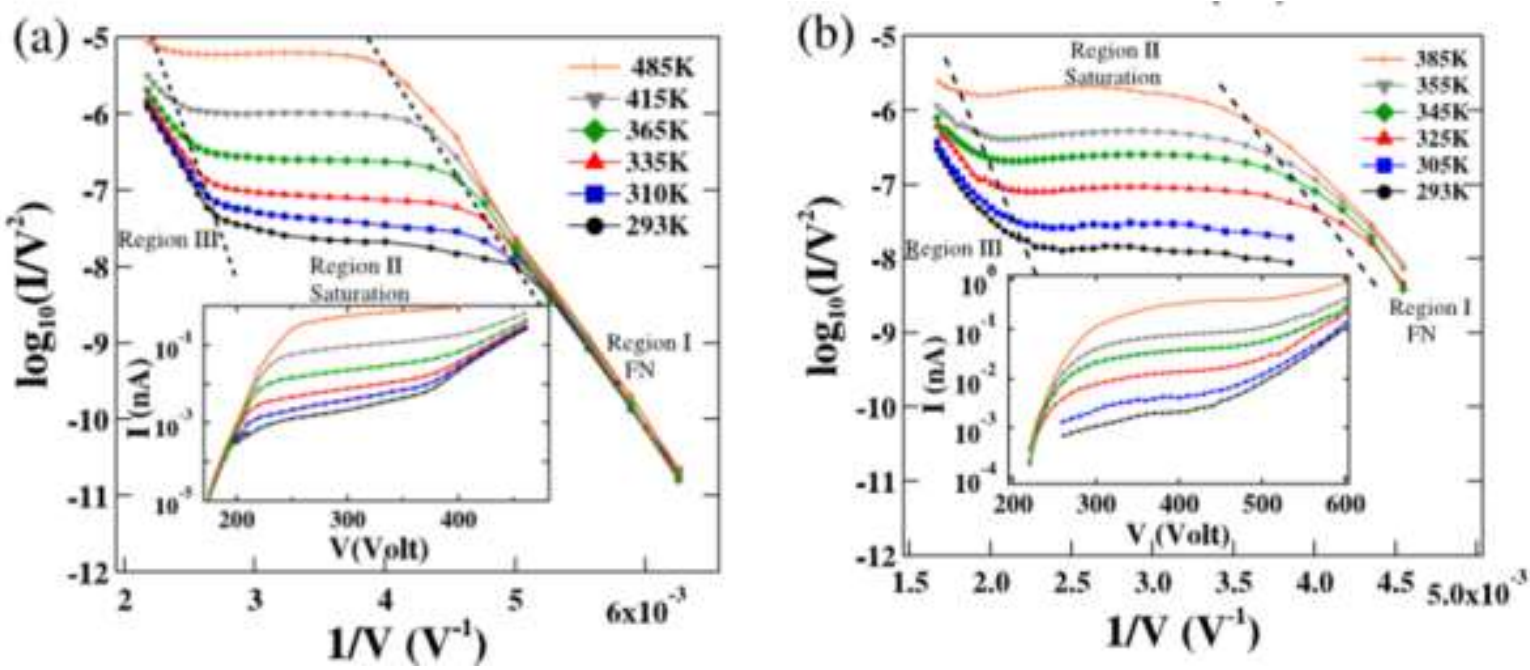

Figure 3. Typical FN plots for individual Si nanowires with increasing temperature: (a) NW1, catalyst end; (b) NW2, substrate end. We did not make use of the electron counter for NW2. One distinguishes the three regions predicted for field emission from semiconductors: Region I: standard FN emission appears at low current $(<1 \mathrm{pA})$ and is measured with the single electron counter. Region II: saturation of current with high sensitivity to temperature. Region III: rapid increase in current. Insets: semilog plots of I(V).

We now explore the use of FE measurements as a tool for understanding the electron transport and for probing the impact of the parameters of the NWs, such as the source of carriers, their concentrations, relaxation times, etc. The equation describing the transport properties of a $\mathrm{p} \_n$ junction where bulk defects and surface states are neglected (low leak current) contains an internal current from GR (IGR), due to the thermal excitation of minority carriers in the SCR, and a diffusion current through the junction (Idiff).16 The saturation current is thus given by: 


$$
\begin{aligned}
I_{\text {sat }} & =I_{G R}+I_{\text {diff }} \\
& =I_{\text {GAO }}\left(\exp \frac{q(\Delta V)}{k T}-1\right)+I_{\text {diffo }}\left(\exp \frac{q(\Delta V)}{2 k T}-1\right)
\end{aligned}
$$

where $\mathrm{q}$ is the elementary charge, $\Delta \mathrm{V}$ is the voltage drop, and $\mathrm{k}$ is the Boltzmann constant. In this expression, IGRo $\sim \mathrm{n}_{\mathrm{i}} / \tau$ and $\mathrm{I}_{\text {diffo }} \sim \mathrm{ni}^{2} / \mathrm{Na}$, where $\tau$ is the minority carrier lifetime, $\mathrm{n}_{\mathrm{i}}$ is the intrinsic carrier concentration, and $\mathrm{Na}_{\mathrm{a}}$ is the concentration of dopants. Because $n_{i} \sim \exp \left(E_{g a p}\right) /(2 k T)$ is relatively small at room temperature for $S_{i}\left(n_{i}=1.45\right.$ $/ \mathrm{cm}_{3}$ ) and $\tau$ is short, 16 IGR ismuch greater than Idiff. Consequently, the carrier transport is dominated by lGR at moderate temperature and can be expressed simply as:

$$
\begin{gathered}
I_{\text {sat }}=I_{G A}=\frac{q n_{i} A W}{2 \tau}\left(\exp \frac{q(\Delta V)}{2 k T}-1\right) \\
=\frac{q\left(N_{c} N_{v}\right)^{1 / 2} A W}{2 \tau}\left(\exp \frac{-E_{g a p}}{2 k T}\right)\left(\exp \frac{q(\Delta V)}{2 k T}-1\right)
\end{gathered}
$$

where $A$ is theNWcross section area,Wis the depletion width across the $p \_n$ junction, $N_{c}$ and $N_{v}$ are the density of states in the valence and conduction bands, respectively.

Equation 2 combined with measurements of the current and voltage drop by the TED (see Experimental Section) allows one to further explore the carrier transport mechanism and intrinsic parameters of the NWs. Consider first the thermal activation energy $\mathrm{E}_{\mathrm{a}}$ of the saturation current in region $\mathrm{II}$ at fixed $\Delta \mathrm{V}$. From eq 2 , it is in principle related to the band gap energy, Egap, of the NWs. However, on the basis of the Shockley_Read_Hall phenomenological theory, this characteristic energy can be lowered by localized trap states in the band gap that serve as GR centers. To evaluate $\mathrm{E}_{\mathrm{a}}$, we have measured I at $\Delta \mathrm{V}=\_30 \mathrm{~V}$ for NW1 and $\Delta \mathrm{V}=\_50 \mathrm{~V}$ for NW2 while changing $\mathrm{T}$, which was done efficiently with a thermocouple attached to the tip mount. Since $\Delta \mathrm{V}$ is large and negative, we can neglect the related exponential term in eq 2, which becomes

$$
I_{\text {sat }}=I_{G A}=\frac{-q\left(N_{c} N_{v}\right)^{1 / 2} A W}{2 \tau} \exp \frac{-E_{s}}{k T}
$$

Note that the current (and also the voltage drop) here is negative, but for simplicity, we used positive values in the plots and in the text hereafter. In Figure 4, we show that $\ln (\mathrm{I})$ versus $1 / \mathrm{kT}$ for NW2 can be very well fitted by the Arrhenius law with $\mathrm{E}_{\mathrm{a}}=0.56 \mathrm{eV}$. This is roughly the $\mathrm{E}_{\mathrm{gap}} / 2$ of $\mathrm{Si}$, which matches the activation energy expected from eq 2. However, we estimated $E_{a}=0.36 \mathrm{eV}$ for NW1, indicating NW to NW differences in activation energies. This is evidenced in Figure 3 by considering that a $150 \mathrm{~K}$ increase for NW1, rather than $80 \mathrm{~K}$ for NW2, is required in order to gain a 2 orders of magnitude change in current. While GR dominated by Au deep traps cannot be ruled out in the case of NW1, their density is probably not enough to completely dominate the GR dynamics in all our NWs. As shown in the following experiments, differences in the surface state density and their role as GR centers explain best the Ea differences in region II between NWs. That is, the higher sensitivity to T for NW2 compared to NW1 is most likely due to a lower concentration of dangling bonds at the NW surface

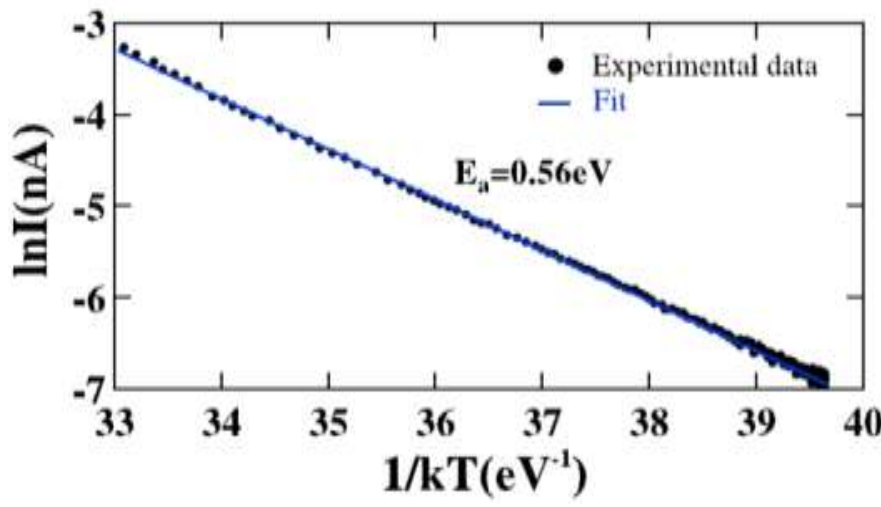

Figure 4. Emission current versus tip temperature for NW2 in the saturation region II and fit with the Arrhenius law with $\mathrm{E}_{\mathrm{a}}=0.56 \mathrm{eV}$. 

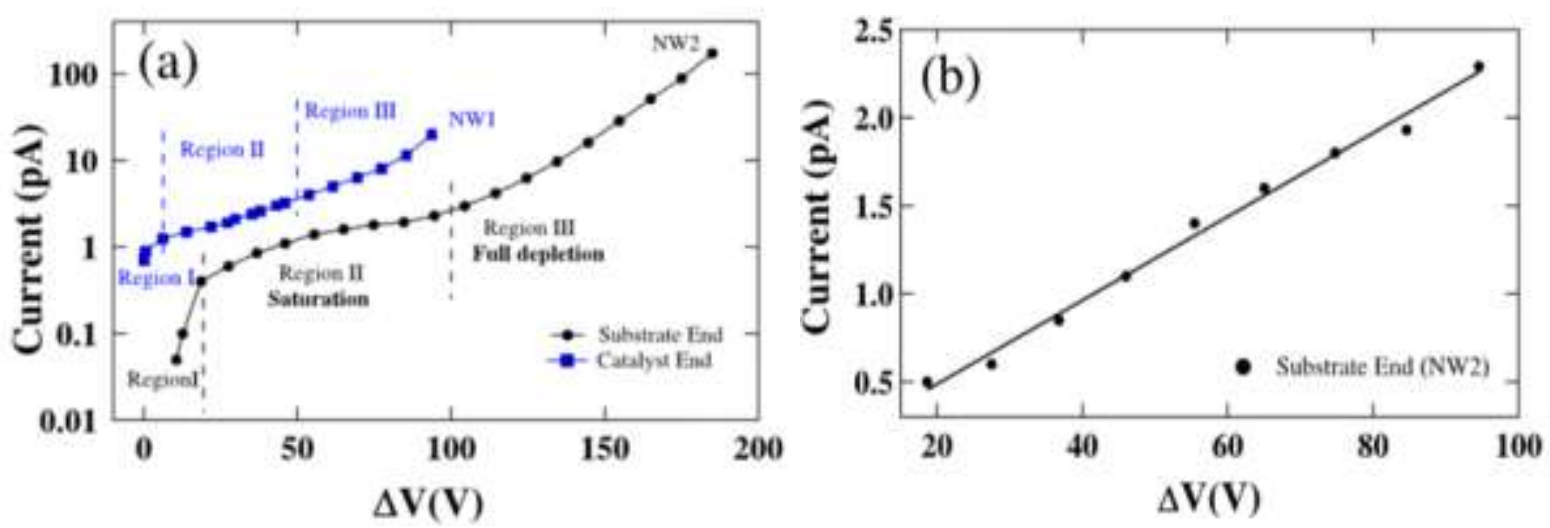

Figure 5. (a) Emission current versus tip voltage drop $\Delta \mathrm{V}$ of NW1-catalyst end and NW2-substrate end emitters. These curves with three different regions are similar in form to $\mathrm{p}_{\mathrm{n}} \mathrm{n}$ junctions in reverse bias. $\Delta \mathrm{V}$ is larger for NW2 than for NW1, and the transition from region II to region III occurs at $\sim 50 \mathrm{~V}$ versus $100 \mathrm{~V}$ for NW2. (b) Emission current versus tip voltage drop of NW2-(substrate end) in the saturation region II, where the linear dependence is clear.

The room temperature I $(\Delta \mathrm{V})$ plots for NW1 and NW2 are shown in Figure $5 a$. These curves are similar in shape to that of a $\mathrm{p} \_n$ junction operating in reverse bias. We can also distinguish the three regions corresponding to the $\mathrm{FN}$ curves of Figure 3. In the linear FN (region I), I is proportional to $\Delta \mathrm{V}$ and the slope gives a resistance of $1013 \Omega$. This value is surprisingly high, roughly $10_{3}$ times greater than the resistance of NWs from the same batch but measured in field-effect transistors (not shown). Therefore, the high resistivity in region I is not a direct measure of the intrinsic resistance of the NWs and rather corresponds to much higher resistance contributions from either the minority carriers (electrons) or from surface carriers accumulated by trap states (i.e., current leak). For region II, we have previously reported that the large voltage drops cannot be explained by the Schottky barrier (SB) at the contact between the W tip and the NWs.1 We can also exclude contact effects due to the oxide because measurements have given low voltage drops ( $2 \mathrm{~V}$ for $20 \mathrm{nA}$ ) for highly p-doped Si NWs with the same oxide thickness (not shown). In addition, the presence of a SB at the Au particle_NW interface can also be neglected for NW1 because an even larger $\Delta \mathrm{V}$ is observed for NW2 while it has no particle. The minor role for the SB found here is consistent with the results by Leonard et al. on Ge NWs.17 Finally, region III presents an increase in current that is characteristic of FN tunneling (i.e., linear in a FN plot). Previously mistaken for a signature of breakdown, it appears clear in our study that this transition is better explained by a SCR that can no longer increase in width because it has reached the maximum length available, which is the full length of the NW. In this respect, it is interesting to note that the transition voltage from region II to region III of NW2 is twice that of NW1 (50 V for NW1 versus $100 \mathrm{~V}$ for NW2), while NW2 is twice as long $(40 \mu \mathrm{m}$ versus $20 \mu \mathrm{m}$ for NW1).

Additional observations in region II can be extracted from the plots in Figure 5a: (i) the $\Delta \mathrm{V}$ for the same I is larger for NW2 than for NW1; (ii) the onset conditions for current saturation are also different: $1 \mathrm{pA}, 1 \mathrm{~V}$ for NW1 versus $0.5 \mathrm{pA}$, $18 \mathrm{~V}$ for NW2; (iii) the slope in the saturation region is larger for NW1 by roughly a factor of 2 . Such differences in FE characteristics are surprising since both NWs have the same diameter and similar dopant density (same batch). At first sight, it may suggest that important screening takes place at the apex because of the presence of the gold particle at the end of NW1. However, a higher surface state density for NW1 is more likely responsible for these differences because only surface traps can affect the $E_{a}$ value and the slope in region II, while also explaining both (i) and (ii) observations. That is, a large density of surface traps can induce carrier inversion at the surface of the NW, which readily creates leak currents for electrons along the NW surface. The larger the leak, the better the screening of the field inside the NW (i.e., lower voltage drops). These hypotheses are tested next using in situ passivation experiments.

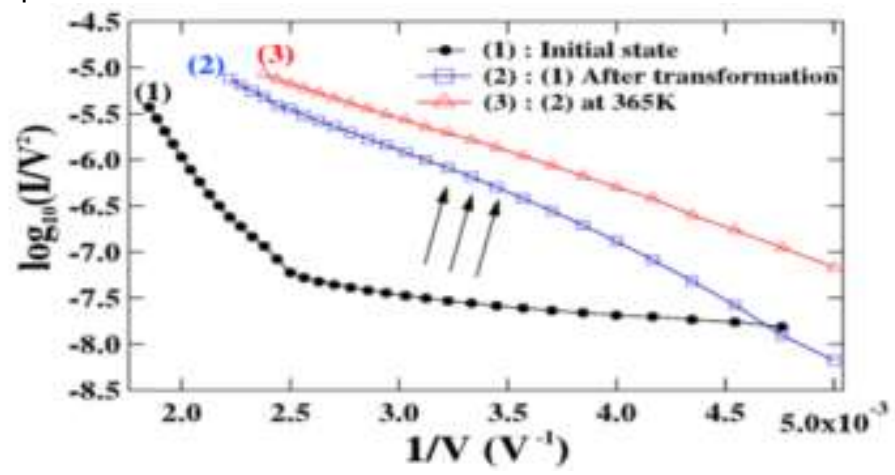

Figure 6. Fowler Nordheim plots for NW1 showing a transformation in emission after laser exposition where the saturation region is less pronounced and the emitted current is less sensitive to temperature (top curves). 
The characteristics established above are for asgrown Si NWs with minimal heating. As shown in Figure 6, a drastic transformation in the FE characteristics was induced by illuminating NW1with a relatively high laser power (7 mW) using a focal spot size of $\sim 20 \mu \mathrm{m}$. This treatment led to the suppression of region II (i.e., saturation). The change is accompanied by a lowering of the resistance by a factor of 100 (not shown). Moreover, the FN I(V) plot in Figure 6 becomes less sensitive to temperature (the blue curve is at room temperature and the red curve at $365 \mathrm{~K}$ ). We have recently explored the effect of such laser heating on other NWs and found that temperature increases in the tens to hundreds of degrees were easily generated in similar structures.18 Extrapolating data in Figure 3, the optical excitation of the Si NW at $7 \mathrm{~mW}$ corresponds to an increase in temperature of $\sim 900 \mathrm{~K}$. This temperature is too low for desorbing the oxide layer, but the interface is expected to be free of all silicon hydride species at $\sim 850$ K.19 Therefore, temperatures induced here by the laser were high enough to eliminate the H-passivation, thereby creating a large concentration of dangling bonds at the $\mathrm{Si} / \mathrm{SiO}_{2}$ interface. Evidently, the heating here has transformed the $\mathrm{H}$-passivated interface into a highly conductive surface "channel", with its overall importance in NW FE probably enhanced due to their high surface/bulk ratios.20
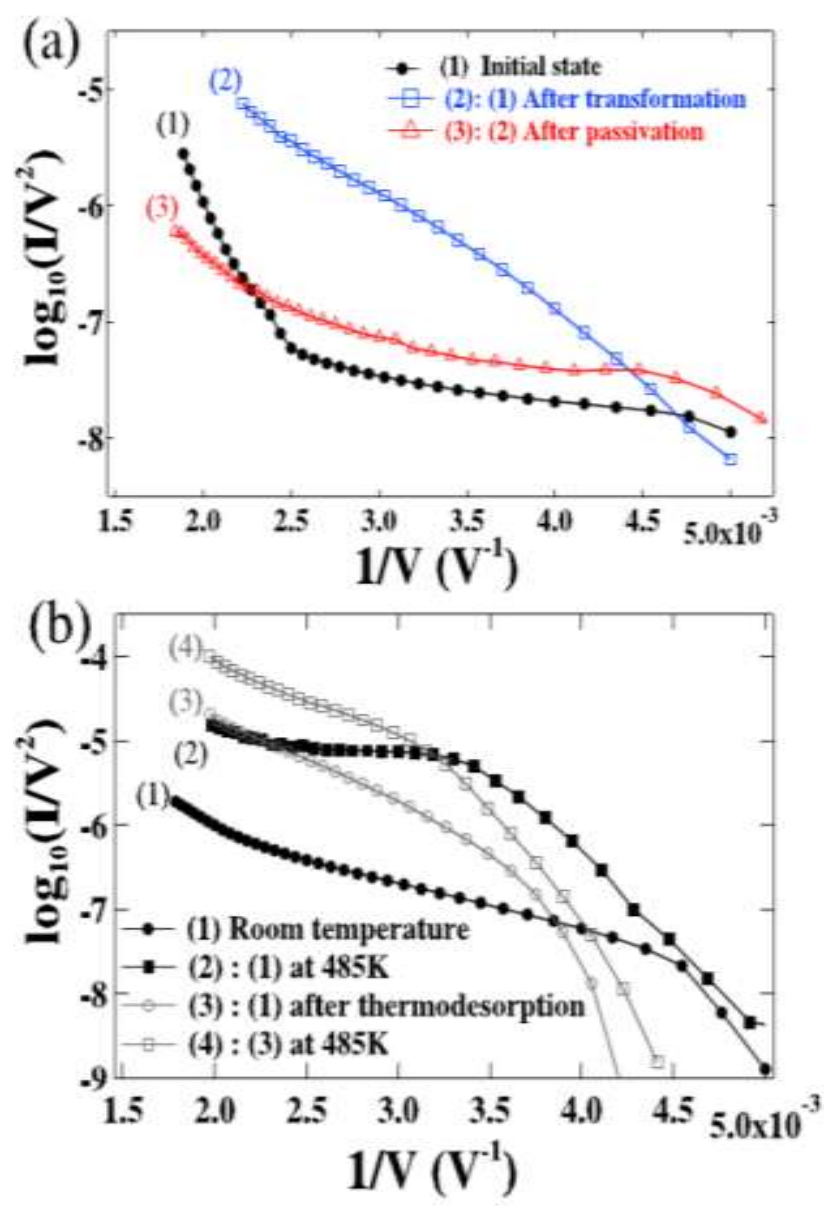

Figure 7. Fowler_Nordheim plots for NW1 with heat- and hydrogen-induced transformations. (a) Before and after the laser-induced transformation and after the hydrogen passivation. NW1 returns almost to the initial state. (b) Effect of thermo-desorption of the hydrogen passivation of NW1 on the FN plots and the sensitivity of current to temperature.

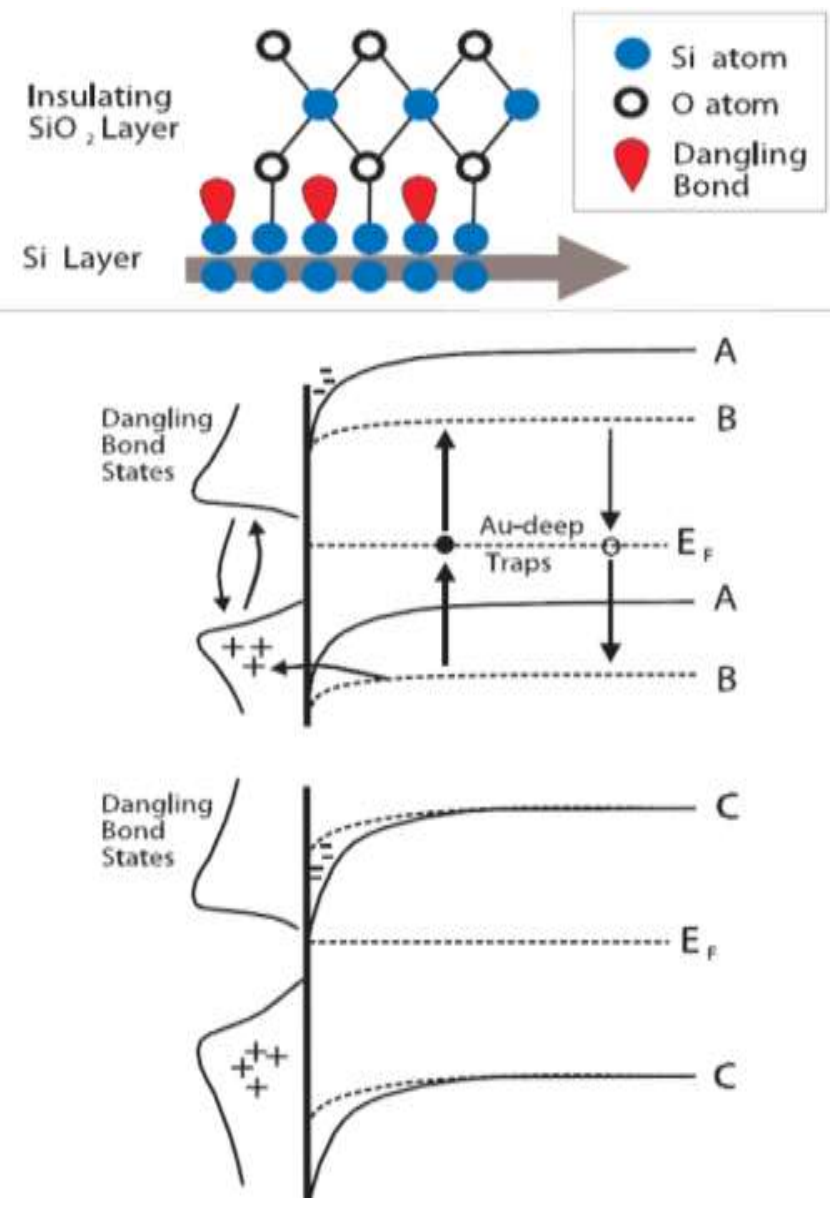

Figure 8. Top: Schemes of the $\mathrm{Si} / \mathrm{SiO}_{2}$ interface of the NW in the presence of positive dangling bonds leading to a conductive inversion layer. Bottom: Band bending situations at the surface of a p-type NW in the presence of positive traps (diagram A) and the same situation but in the SCR induced by the electric field (diagram B). The influence of an increase of trap density leading to a pinning of the Fermi level to the conduction band as illustrated in diagram C. Also illustrated are the generation recombination processes involving $A u$ deep traps in the SCR, the hole trapping mechanism, and the electron recombination at the surface involving trapped holes.

In order to reproduce the initial characteristics, an in situ passivation step was carried out. It is common in Si technology to include a step aimed at passivating dangling bonds and other trap states at the $\mathrm{Si}_{/} \mathrm{SiO}_{2}$ interface using 
annealing in a forming gas containing hydrogen.21 Hydrogen atoms can diffuse through the $\mathrm{SiO}_{2}$ layer toward the interface and react with Si dangling bonds and other low coordinated $\mathrm{Si}$ atoms in the $\mathrm{SiO}_{2}$. This has been used for $\mathrm{Si}$ NWs in the transistor configuration,22,23 though we have not found an in-depth study that correlates such hydrogen passivation with a modification of the transport properties. As shown in Figure 7, atomic hydrogen exposure was effective to passivate the NWs in the FE configuration in vacuum (see Experimental Section). FN plots for NW1 taken before and after passivation are shown in Figure 7a. These results confirm our hypothesis that dangling bonds can lead to a significant surface leak that alters the FE characteristics of SCs. This behavior was reproduced through successive and gradual thermodesorption (TD)/passivation cycles showing how the conduction properties could be efficiently tuned. A variation of the average resistance by 2 orders of magnitude was achieved. For the TD, the NW was heated for different times to $870 \mathrm{~K}$ to desorb the passivating $\mathrm{H}$ at the $\mathrm{Si} / \mathrm{SiO}_{2}$ interface. Figure $7 \mathrm{~b}$ shows the result for one cycle of TD/passivation represented in FN curves. As expected, the sensitivity of I to T is much higher for the best passivated NWs. The in situ passivation was in all cases incomplete as the NWs never recovered completely the pristine state measured after the growth. Thismight be due to the high directionality of the atomic beam of hydrogen atoms generated in vacuum from the filament located in front of the NW.

The in situ passivation experiments in Figure 7 have shown that an extra conduction channel is formed in parallel when the NWs have a large number of dangling bonds at the $\mathrm{Si} / \mathrm{SiO}_{2}$ interface. What is the microscopic nature of this extra channel for the current? The Si dangling bonds are known to be good electron donors relative to Si bulk.24 As illustrated in Figure 8, they produce surface traps that are positive and thus induce, when in significant number, a band bending and a depletion of holes near the NW surface (diagram A). When the trap density is large enough, the band bending leads to an electron accumulation thatforms an extra conductive channel along the NW but at the surface only. In the extreme case, a metallic channe can even be formed at the surface,24 which may completely eliminate the saturation in region II (diagram $\mathrm{C}$ ).

DISCUSSION

The saturation behavior is next discussed for NW2 using eq 3 at room temperature $\left(\mathrm{n}_{\mathrm{i}}=1.45 \_10_{10} / \mathrm{cm}_{3}\right)$ :

$$
l_{s t}=\frac{q n_{1} A W}{2 \tau}
$$

Because I is proportional to $\mathrm{W}$ in the saturation region, the linear fit of I versus $\Delta \mathrm{V}$ in Figure $5 \mathrm{~b}$ for NW2 thus implies that $W_{-} V$. This linear dependence contrasts with the $W_{\sim} V^{1 / 2}$ for bulk diodes. A deviation from the root square law is, however, not surprising for lowdimensional Si NWs. On one hand, Léonard et al. have shown that W can follow an exponential dependence on reverse bias in Ge Schottky diode NWs.17 On the other hand, and consistent with our results, a linear dependence of $\mathrm{W}$ has been predicted in 2D p_n junctions 25 and observed in devices made of NW diodes.26,27 The expression given for $\mathrm{W}$ is 25

$$
W=k \Delta V=\frac{\varepsilon_{1} \varepsilon_{0}}{2 \pi q N_{5}} \Delta V
$$

where $\varepsilon_{r}$ is the relative dielectric constant of $\mathrm{Si}, \varepsilon_{0}$ is the vacuum permittivity and $\mathrm{Ns}$ is the surface doping concentration. Because of the linear dependence, the average electric-field strength in the $p \_n$ junction depends only weakly on the external bias. This unusual behavior allows an extended SCR where the breakdown voltage shifts to very high reverse biases.26,27 As a result, the depletion region can be very large for $\mathrm{FE}$, as demonstrated theoretically4 and experimentally for macroscopic emitters 3 and NWs.2 We therefore conclude that the transition from region II to III is not due to breakdown, but rather marks a transition when the SCR is extended to the full length of the NWs. We can then estimate $\mathrm{k}$ for NW2 using

$$
k=\frac{W_{\max }}{\Delta V_{\mathrm{t}}}=\frac{L}{\Delta V_{\mathrm{t}}}=0.4 \mu \mathrm{m} / \mathrm{V}
$$

This relation gives an estimation of $\mathrm{N}_{\mathrm{s}}$ and then of $\mathrm{N}_{\mathrm{A}}$ using $\mathrm{N}_{\mathrm{A}}=\mathrm{N} \mathrm{s} / \mathrm{d} \sim 2 \sim 10^{15} / \mathrm{cm}^{3}$. Using the same approach, the same doping was also estimated for NW1.Assuming no leakage current for NW2, a carrier lifetime $\tau=66$ ps is readily extracted from the slope of the line in Figure $5 \mathrm{~b}$ and eq 4. This low value compared to bulk indicates that the rate is probably dominated by surface recombination, which is expected for NWs because of their large surface-to volumeratio. The lifetime in such case is given by28,29

$$
\frac{1}{\tau_{\mathrm{NW}}}=\frac{1}{\tau_{\mathrm{bulk}}}+\frac{4 S}{d}
$$

where $\tau \mathrm{NW}$ ( $\tau_{\text {bulk }}$ ) represents the NW (bulk) carrier lifetime, $\mathrm{d}$ is the diameter of NW, and $\mathrm{S}$ is the surface velocity. The total lifetime is reduced by the surface term $(4 \mathrm{~S} / \mathrm{d})$. In theNWliterature, the bulk term is known to be negligible compared to the surface term.30 Indeed, it has been demonstrated that the influence of incorporated impurities on the electronic properties of Si NWs is insignificant,30 even for a low density of surface states.28 The above expression can therefore be written as: 


$$
\frac{1}{\tau_{\mathrm{NW}}}=\frac{4 \mathrm{~S}}{d}
$$

and gives $\mathrm{S}=4 \ldots 10^{4} \mathrm{~cm} / \mathrm{s}$, which is extremely large compared to $\mathrm{H}$-terminated flat $\mathrm{Si}$ surfaces 31 but physically reasonable for NWs.29,30 Finally, we can estimatethe density of recombination centers at the surface $\mathrm{Nr}$ from the Shockley_Read_Hall expression:16

$$
S=v_{\text {th }} \sigma N_{\mathrm{r}}
$$

where $\mathrm{Vth}_{\mathrm{th}}(\mathrm{Si}) \sim 2 \_10^{7} \mathrm{~cm} / \mathrm{s}$ is the mean thermal velocity of carriers and $\sigma(\mathrm{Si})=10^{-15} \mathrm{~cm}^{2}$ is their mean capture cross section.32 We obtain $\mathrm{Nr}=1.7 \quad 10^{12} / \mathrm{cm}^{2}$, which is about an order of magnitude lower than $\mathrm{Si} / \mathrm{SiO}_{2}$ interface state density for native oxides.30 This proves that the passivation achieved during growth reduced significantly the surface state density and contributed to the strong saturation in FN curves. However, this density is greater than the values reported for Si NWs passivated at high temperature using thermal oxidation and a forming gas anneal $\left(\mathrm{N}_{r}=5 \sim 10\right.$ $10^{11} / \mathrm{cm}^{2}$ ) for Toxidation $=850 \_\mathrm{C}_{23}$ and $\left.\mathrm{Nr}_{r}=5 \sim 10 \% \mathrm{~cm}^{2}\right)$ for Toxidation $=960{ }^{\circ} \mathrm{C}_{28}$ ). The considerably higher density found here can be attributed to the modest quality of our oxide obtained during the low temperature growth (540 ${ }^{\circ} \mathrm{C}$ ). Forming a high-quality oxide on the NWs surface using an additional oxidation step at higher temperature followed by standard hydrogen passivation can therefore be effective in further improving the saturation.

In this model, the influence of surface states in the SCR appears mainly as a competitive GR channel for reducing the carrier lifetime. The interplay between the GR processes in the bulk, on Au deep traps, and at the surface thus appears in the analysis as the main factor determining the current and the slope in region II. This is, however, valid only if the leak current is small. This condition for the SCR is illustrated in Figure 8, diagram B.

When the density of surface states is extremely large, an extra conduction channel is formed at the surface of the NWdue to a significant shift of the pinning position of the Fermi level at the $\mathrm{Si} / \mathrm{SiO}_{2}$ interface24 (diagram $\mathrm{C}$ of Figure 8 ). This leads to a large current leakage and a complete loss of region II, such as in Figure 7a. Therefore, the role of the surface states is not only to control the GR processes but also to induce a leak current, which is a competitive mechanism acting in parallel with Isat. In the low-field region (region I), leak can be estimated using lleak = $\left(\mathrm{A}_{0} / \mathrm{L}\right) q n_{i t} \mu_{\mathrm{e}} \Delta \mathrm{V}$, where $\mathrm{n}_{\mathrm{t}}$ is the density of the electron layer at the surface induced by the hole traps, $\mu \mathrm{e}$ is their mobility, $\Delta \mathrm{V}$ the internal voltage drop, and $\mathrm{L}$ and $\mathrm{A} 0$ are the NW length and section for this electron layer. This leak resistance is, however, difficult to estimate experimentally because the currents are very low and most often below the sensitivity of the electron analyzer. In general, the leakage current should dominate when $\mathrm{nit}_{\mathrm{i}}>\mathrm{ni}$.

When the passivation is only partial, the situation is more complex because it involves a competition between lieak and Isat. In this case, it is interesting to note that low leak affects weakly the estimation of $\tau$ from the slope in region II. For instance, Rleak for NW2 is very large $\left(10^{13} \Omega\right)$ and leak is negligible and has little effect on the estimate of GR rate. It is also important to keep in mind that the trapped holes at the surface will accelerate the recombination dynamics in the SCR. Thus, the very fast recombination process for NW2 is a clear indication that the passivation in this case can be further improved. However, a perfectly passivated NW would appear here as a bad choice for applications because it will produce uselessly small Isat. Similar arguments can also be invoked in order to optimize the temperature response in FE by simple adjustments of the $E_{a}$ value of the NWs through a tight control on their surface passivation.

\section{CONCLUSION}

In this paper we studied FE from individual Si NWs. Quasi-ideal saturation was obtained accompanied by a strong sensitivity to temperature. The highest sensitivity was an increase in current by 2 orders of magnitude for an increase in T of only $80 \_$C. Furthermore, the measurements of the TEDs of the emitted electrons and a simple p_n junction model taking into account the low dimensionality of the NWs allowed us to estimate the doping and carrier lifetime. The latter was found to be dominated by surface recombination. The role of the surface states was demonstrated by cyclic heating and hydrogen passivation treatments done in situ. This treatment allowed us to determine that the current saturation is intimately linked to the good conducting properties of the $\mathrm{Si} / \mathrm{SiO}_{2}$ interface of the NWs. An important point is that until nowmodifications of the FE from SCs were considered as irreversible.

In general, a more quantitative understanding and control of the emission phenomena from SC NWs may now be achievable. These strong saturation effects must be associated with the high quality of the Si NWs, and the results now appear solid enough to be of interest for a more rigorous theoretical treatment of the problem. Such a treatment is simplified by the uniform cross section of the NWs as compared to the more frequently encountered conic emitters. However, self-consistent solutions that marry SC transport theory, FE, three dimensions, and eventually the nanometric effects such as confinement must be sought. In the past years, the NW community has succeeded in mastering the fabrication and analysis of ever higher quality and more complex NW structures. The methodology exposed here is thus applicable to a rich panoply of systems and phenomena. 
Riber e-beam evaporator. The synthesis was carried out in a previously described homemade hot-filament-assisted CVD system at 540 C. 33,34 The $0.38 \mathrm{~mm}$ diameter tungsten wire, mounted at the $\mathrm{H}_{2}$ feedthrough inlet, was powered at $175 \mathrm{~W}$, corresponding to an approximate surface temperature of 1900 _C. Efficient $\mathrm{H}_{2}$ decomposition was thus achieved by forcing it to pass through the hot wire confined within a thermal screen. The provided atomic hydrogen flow acted as an efficient etchant to avoid undesired amorphous $\mathrm{Si}$ (a-Si) deposition during $\mathrm{Si}$ NW growth. After temperature ramping to 540 _C (20_C per minute) and prior to the Si NW growth, the samples were exposed to hotfilament-activated hydrogen flow for $5 \mathrm{~min}$. This was done to remove eventual contaminants and to achieve efficient dewetting of the gold thin film and the formation of the catalyst particles onto which $\mathrm{Si}$ NWs nucleated and grew. Si NW growth was then initiated by silane $\left(\mathrm{SiH}_{4}\right)$ precursor gas injection at 10\% dilution in hydrogen for a total pressure of 15 mbar and a total flow of $100 \mathrm{sccm}$. During the 30 min growth time, the hot filament was continuously powered at $175 \mathrm{~W}$

For FE studies, individual NWs were attached directly from the growth substrate to the ends of tungsten tips that were previously sharpened by electrochemical etching in $\mathrm{NaOH}$ solution. The attachment was done through conductive carbon glue, and positioning was achieved using a 3D micromanipulator and an optical microscope. The NWs were first selected using the coated tip via van der Waals forces and then detached from the substrate by pulling along the tip axis.

Experimental Setup. FE measurements were performed in an UHV system (2_10_10 Torr) that has been described in detail elsewhere.1,2,18 The polyvalent setup allows one to carry out a wide range of measurements. An electron counter permits low current measurements starting at 10_20 A. The temperatures can be varied simultaneously from 100 to $2000 \mathrm{~K}$. It also has a home-built hot filament system for ion bombardment and the decomposition of neutrals useful for surface treatments with different gases. For optical studies, an Ar laser is focused onto the field emitter with a standard optical setup mounted outside the vacuum chamber in front of a viewport. Finally our installation has the great advantage of having an electron energy analyzer for direct measurements of the TEDs. The TEDs give the Fermi level at the NW apex with respect to that of the support tip and thus the voltage drop $\Delta \mathrm{V}$ along the NW. These measurements combined with the measured total current become a type of two-point transport measurement to study the carrier transport mechanisms in individual SiC NWs.2

Passivation. $\mathrm{H}_{2}$ flowing at a pressure of $10 \_4$ Torr was dissociated by a hot tungsten filament positioned in the FE chamber near the NW (2000 _C measured by an optical pyrometer) for several minutes.35 The NW was held at room temperature. This short treatment was sufficient to recover almost completely the current saturation and region II.

Acknowledgment. This work was carried out within the framework of the "Plateforme nanofils et nanotubes lyonnaise". Financial support for this work is from the CRSNG Discovery and Canada Research Chair grants.

\section{REFERENCES AND NOTES}

1. Choueib, M.; Ayari, A.; Vincent, P.; Bechelany, M.; Cornu, D.; Purcell, S. T. Strong Deviations from Fowler Nordheim Behavior for Field Emission from Individual SiC Nanowires Due to Restricted Bulk Carrier Generation. Phys. Rev. B 2009, 79, 075421.

2. Choueib, M.; Ayari, A.; Vincent, P.; Perisanu, S.; Purcell, S. T. Evidence for Poole-Frenkel Conduction in Individual SiC

Nanowires by Field Emission Transport Measurements

J. Appl. Phys. 2011, 109, 073709.

3. Arthur, R. J. Photosensitive Field Emission from p-Type

Germanium. J. Appl. Phys. 1965, 36, 3221-3227.

4. Baskin, L. M.; Lvov, O. I.; Fursey, G. N. General Features of

Field Emission from Semiconductors. Phys. Status Solidi B

1971, 47, 49-62.

5. Fursey, G. N.; Egorov, N. V. Field Emission from p-Type Si.

Phys. Status Solidi B 1969, 32, 23-29.

6. Schroder, D. K.; Thomas, R. N.; Vine, J.; Nathanson, H. C. The

Semiconductor Field-Emission Photocathode. IEEE Trans.

Electron Devices 1974, 21, 785-798.

7. Fursey, G. N. Field Emission in Vacuum Microelectronics;

Academic Press: New York, 2005; pp 77_78.

8. Liu, K. X.; Chiang, C. J.; Heritage, J. P. Photoresponse of

Gated P-Silicon Field Emitter Array and Correlation with

Theoretical Models. J. Appl. Phys. 2006, 99, 034502.

9. Sawada, K.; Ishida, M. Novel Photocathodes Using Vacuum

Microelectronics Technology. Sens. Actuators, A 2000, 82,

259-264.

10. Zeng, B.; Xiong, G.; Chen, S.; Jo, S. H.; Wang, W. Z.; Wang,

D. Z.; Ren, Z. F. Field Emission of Silicon Nanowires. Appl.

Phys. Lett. 2006, 88, 213108.

11. Pimenov, S. M.; Frolova, V.D.; Kudryashovb, A. V.; Lamanovb,

M. M.; Abanshinc, N. P.; Gorfinkelc, B. I.; Kimd, D.-W.; Choid Y.-J.; Parkd, J.-H.; Parkd, J.-G. Electron Field Emission From Semiconducting Nanowires. Diamond Relat. Mater. 2008,

$17,758-763$

12. Zeng, H.; Xu, X.; Bando, Y.; Gautam, U. K.; Zhai, T.; Fang, $\mathrm{X}$;

Liu, B.; Golberg, D. Template Deformation-Tailored ZnO

Nanorod/Nanowire Arrays: Full Growth Control and Optimization

of Field-Emission. Adv. Funct. Mater. 2009, 19,

3165-3172.
13. Datta, A.; Chavan, P. G.; Sheini, F. J.; More, M. A.; Joag, D. S.;

Patra, A. Growth, Optical, and Field Emission Properties of Aligned CdS Nanowires. Cryst. Growth Des. 2009, 9, 41574162.

14. Eichfeld, S. M.; Ho, T. T.; Eichfeld, C. M.; Cranmer, A.; Suzanne, E.; Mohney, S. E.; Mayer, T. S.; Redwing, J. M.

Resistivity Measurements of Intentionally and Unintentionally Template-Grown Doped Silicon Nanowire Arrays.

Nanotechnology 2007, 18, 315201.

15. Tavendale, A. J.; Pearton, S. J. Deep Level, Quenched-In Defects in Silicon Doped with Gold, Silver, Iron, Copper or Nickel. J. Phys. C: Solid State Phys. 1983, 16, 1665-1673. 16. Sze, S. M. Physics of Semiconductor Devices; Wiley Interscience:

New York, 1969; pp 96_104.

17. Leonard, F.; Talin, A. A.; Swartzentruber, B. S.; Picraux, S. T. Diameter-Dependent Electronic Transport Properties of Au-Catalyst/Ge-Nanowire Schottky Diodes. Phys. Rev. Lett. 2009, 102, 106805.

18. Choueib, M.; Ayari, A.; Poncharal, P.; Journet, C.; Cojocaru, C. S.; Martel, R.; Purcell, S. T. Field Emission Measure of the Time Response of Individual Semiconducting Nanowires to Laser Excitation. Appl. Phys. Lett. 2011, 99, 072115. 19. Sun, X. H.; Wang, S. D.; Wong, N. B.; Ma, D. D. D.; Lee, S. T.;

Teo, B. K. FTIR Spectroscopic Studies of the Stabilities and Reactivities of Hydrogen-Terminated Surfaces of Silicon Nanowires. Inorg. Chem. 2003, 42, 2398-2404.

20. Fernandez-Serra, M. V.; Adessi, C.; Blase, X. Conductance,

Surface Traps and Passivation in Doped Silicon Nanowires.

Nano Lett. 2006, 6, 2674-2678.

21. Ibach, I.; Rowe, J. E. Hydrogen Adsorption and Surface

Structures of Silicon. Surf. Sci. 1974, 43, 481-492.

22. Cui, Y.; Zhong, Z.; Wang, D.; Wang, W. U.; Lieber, C. M. High

Performance Silicon Nanowire Field Effect Transistors.

Nano Lett. 2003, 3, 149-152.

23. Seo, K.; Sharma, S.; Yasseri, A. A.; Stewart, D. R.; Kamins, T. I.

Surface Charge Density of Unpassivated and Passivated

Metal-Catalyzed Silicon Nanowires. Electrochem. Solid-

State Lett. 2006, 9, G69-G72.

24. Luth, H. Surfaces and Interfaces of Solid Materials; Springer 
study edition, 1995; pp 381_391.

25. Achoyan, A. S.; Yesayan, A. E.; Kazaryan, E. M.; Petrosyan,

S. G. Two-Dimensional p_n Junction under Equilibrium

Conditions. Semiconductors 2002, 36, 903-907.

26. Hayden, O.; Zheng, G.; Garwal, P.; Lieber, C. M.

Visualization

of Carrier Depletion in Semiconducting Nanowires. Small

2007, 3, 2048-2052.

27. Hwang, J.-S.; Donatini, F.; Pernot, J.; Thierry, R.; Ferret, P.;

Dang, L. S. Carrier Depletion and Exciton Diffusion in a

Single ZnO Nanowire. Nanotechnology 2011, 22, 475704.

28. Demichel, O.; Calvo, V.; Besson, A.; Noe, P.; Salem, B.;

Pauc,

N.; Oehler, F.; Gentile, P.; Magnea, N. Surface Recombination

Velocity Measurements of Efficiently Passivated Gold-

Catalyzed Silicon Nanowires by a New Optical Method.

Nano Lett. 2010, 10, 2323-2329.

29. Dan, Y.; Seo, K.; Takei, K.; Meza, J. H.; Javey, A.; Crozier, K.

B.

Dramatic Reduction of Surface Recombination by In Situ

Surface Passivation of Silicon Nanowires. Nano Lett. 2011,

11, 2527-2532.

30. Allen, J. E.; Hemesath, E. R.; Perea, D. E.; Lensch-Falk, J.

$\mathrm{L} . ; \mathrm{Li}$,

Z. Y.; Yin, F.; Gass, M. H.; Wang, P.; Bleloch, A.; et al. High-

Resolution Detection of Au Catalyst Atoms in Si Nanowires.

Nat. Nanotechnol. 2008, 3, 168-173.

31. Yablonovitch, E.; Allara, D. L.; Chang, C.; Gmitter, T.; B, B. T.

Unusually Low Surface-Recombination Velocity on Silicon

and Germanium Surfaces. Phys. Rev. Lett. 1986, 57, 249-

252.

32. Hull, R. Properties of Cristalline Silicone; INSPEC

Publication:

London, 1999; pp 569_570.

33. Jeong, H. J.; Eude, L.; Gowtham, M.; Marquardt, B.; Lim, S.

$\mathrm{H}$;

Enouz, S.; Cojocaru, C. S.; Lee, Y. H.; Pribat, D. Atomic

Hydrogen Pretreatment for Low Temperature Growth of

Single-Walled Carbon Nanotubes. Nano 2008, 3, 145-153.

34. Lefeuvre, E.; Kim, K. H.; Chatelet, M.; Pribat, D.; Cojocaru,

C. S. Optimization of Organized Silicon Nanowires Growth

Inside Porous Anodic Alumina Template Using Hot Wire

Chemical Vapor Deposition Process. Thin Solid Films 2011,

519, 4603-4608.

35. Langmuir, I. The Dissociation of Hydrogen into Atoms.

J. Am. Chem. Soc. 1912, 34, 860-877. 